\title{
Effects of response experience and concurrent hypothalamic stimulation on electrically elicited behavior
}

\author{
DRAKE C. CHISHOLM \\ Bridgewater State College, Bridgewater, Massachusetts 02324 \\ and
}

JAY A. TROWILL

University of Denver, Denver, Colorado 80210

\begin{abstract}
Rats that received specific drinking experience during electrical stimulation of the lateral hypothalamus (ESLH) were tested to see if the type of behavior to be induced by subsequent ESLH could be influenced. The results indicated that the initial behaviors evoked by ESLH are not affected by a previous contiguity between stimulation and response. Although electrically induced behaviors are sensitive to internal states and external stimuli, these factors have a minimal effect on the type of behavior that initially emerges.
\end{abstract}

Information concerning the anatomical location of electrodes has not been sufficient to predict the type of behavior elicited by electrical stimulation of the lateral hypothalamus (ESLH; Valenstein, Cox, \& Kakolewski, 1969). Therefore, attempts to determine the likelihood of a particular behavior being elicited by ESLH have focused on certain environmental factors which may affect the relative strength of a response in an animal's response hierarchy during the initial stimulation session (Valenstein, Cox, \& Kakolewski, 1970).

The most direct attempts to manipulate the strength of a response in an animal's hierarchy have tried to associate a particular motivational state and response with specific environmental stimuli (Valenstein \& Cox, 1970). However, many of these tests involved attempts to control previously established stimulus-bound behavior and did not investigate the type of response to initially occur. When naive animals were used, a strict contiguous relationship between the performance of the consummatory response and the delivery of the ESLH was not maintained. Therefore, the present study used naive, nondeprived animals and presented ESLH in strict contiguity with the performance of the consummatory response so that it could be determined whether the combined effect of previous experience contiguous with ESLH was sufficient to later affect the type of stimulus-bound behavior to emerge.

Requests for reprints should be sent to D. C. Chisholm, Department of Psychology, Bridgewater State College, Bridgewater, Massachusetts 02324 .

\section{METHOD}

Subjects

Ten Charles River albino rats, approximately 90-100 days old, were used. The animals were housed under constant lighting conditions and were allowed free access to food (Purina Lab Chow) and water in the home cages.

\section{Surgery}

Each rat was anesthetized with Nembutal anesthesia $(40 \mathrm{mg} / \mathrm{kg})$ and bilaterally implanted with stainless steel monopolar electrodes insulated except for $.5 \mathrm{~mm}$ at the tips. The electrodes were stereotaxically placed $.8 \mathrm{~mm}$ posterior to bregma, $1.7 \mathrm{~mm}$ lateral to the midline, and $8.9 \mathrm{~mm}$ below the top of the skull (Pellegrino \& Cushman, 1967). The mouth bar was set $5 \mathrm{~mm}$ above the interaural line.

\section{Apparatus}

A $30 \times 30 \times 45 \mathrm{~cm}$ fiberboard box served as the experimentäl chamber. Two $71 / 2-\mathrm{W}$ light bulbs and a $6-\mathrm{cm}$ speaker delivering $70 \mathrm{~dB}$ of white noise were located directly over the box. On one side of the chamber, two metal drinking spouts were recessed in Plexiglas shields so that discrete tongue contacts could be recorded via electronic drinkometers. The electrical stimulation for all phases of the experiment was a 60-cycle sine wave. The current was regulated by an ac micropotentiometer and was continuously monitored by a cathode-ray oscilloscope. The presentation and recording of events was automated through the use of conventional programming equipment.

\section{Procedure}

Training. Five to 7 days following surgery, each animal was placed in the empty experimental chamber and was given 20 trials per day for 4 days, during which it was exposed (in the absence of stimulation) to a two-bottle choice situation consisting of water or a $12 \% \mathrm{w} / \mathrm{w}$ sucrose solution. Each trial was composed of a 30-sec period during which the drinking tubes were introduced and a 60-sec interstimulus interval during which the tubes were withdrawn. A cam motor was used to automatically insert and withdraw the tubes. Half of the animals always received the sucrose solution on the left side of the apparatus, while the remainder of the animals received it on the right side. 
Table 1

Mean Lick Rate Per Minute for Days 7-9 of Training

\begin{tabular}{crrrrrrrr}
\hline & \multicolumn{2}{c}{ Day 7} & & \multicolumn{2}{c}{ Day 8} & & \multicolumn{2}{c}{ Day 9 } \\
\cline { 2 - 3 } \cline { 7 - 8 } Subject & Water & Sucrose & & Water & Sucrose & & Water & Sucrose \\
\hline 1 & 0.0 & 141.0 & & 0.7 & 180.0 & & 0.3 & 192.0 \\
2 & 0.3 & 156.7 & & 1.0 & 177.0 & & 0.2 & 187.0 \\
3 & 21.0 & 85.7 & & 0.5 & 144.0 & & 0.0 & 154.0 \\
4 & 0.5 & 194.0 & & 0.9 & 157.0 & & 1.0 & 141.0 \\
5 & 0.0 & 186.0 & & 1.0 & 166.0 & & 0.0 & 172.0 \\
6 & 0.0 & 112.0 & & 0.7 & 170.0 & & 0.6 & 123.0 \\
7 & 0.0 & 147.0 & & 0.5 & 138.0 & & 0.0 & 135.0 \\
8 & 0.0 & 191.0 & & 2.0 & 179.0 & & 0.0 & 154.0 \\
9 & 0.0 & 69.0 & & 0.0 & 108.0 & & 0.0 & 105.0 \\
10 & 3.5 & 164.0 & & 0.5 & 167.0 & & 0.0 & 153.0 \\
\hline
\end{tabular}

During Training Days 5-9, both the sucrose and the water spouts were presented, and, after the animal made five rapid contacts with either tube, the stimulation was switched on. Thirty seconds later, the drinking tubes were withdrawn simultaneously with the offset of the stimulation. Each day during this phase of the experiment, the current intensity was slowly increased until the drinking was disrupted as indicated by a tendency for the animal to break contact with the tube for $2-3 \mathrm{sec}$. Then the current intensity was immediately decreased by 2-3 microA or until consistent drinking behavior was once again observed. In this way, the electrical stimulation was maintained at its maximum intensity while the animal was drinking. Each daily session was composed of 30 stimulation trials of $30 \mathrm{sec}$ duration with a 60 -sec interstimulus interval. Three large Purina Lab Chow pellets and a soft pine wedge, $5 \times 5 \times 5 \mathrm{~cm}$, were in the chamber throughout the last 5 training days.

Following training, all animals were given 5 days of testing, during which all conditions remained the same as during training, except that both the water and sucrose were continuously available and the ESLH came on for $30 \mathrm{sec}$ with a 60 -sec interstimulus interval independent of the animal's behavior. Thus the animals were tested to determine if the previous experience with a response plus the concurrent delivery of ESLH would produce a higher percentage of stimulus-bound sucrose drinkers than gnawers, eaters, or water drinkers.

\section{RESULTS}

\section{Histology}

Following data collection, the animals were sacrificed for histological verification of electrode placement. Each animal was given an overdose of Nembutal anesthesia followed by perfusion with $10 \%$ Formalin. The brains were frozen, and 90 -micron frontal sections were stained with cresyl violet and mounted on glass slides. In all cases, the electrodes were located in the lateral hypothalamus or zona incerta similar to the placements reported earlier (Chisholm \& Trowill, 1972; Valenstein, Cox, \& Kakolewski, 1970).

\section{Training}

During the first 4 days of training, observations indicated that all animals learned to drink the sucrose solution for the full 30-sec periods. On Day 5, when the ESLH was faded in during the drinking periods, the response was not disrupted. Table 1 presents the mean lick rate per minute for Days 7-9 of training. Although many animals sampled the water at some time during the training sessions, the dominant response concurrent with the ESLH was sucrose drinking.

\section{Testing}

Due to the fact that none of the animals drank water during testing, just the sucrose data are presented in Table 2. Stimulus-bound behavior is indicated by a high lick rate during the electrical stimulation period and a low lick rate during the interstimulus interval. In spite of the fact that all animals had 230 training trials with the drinking response and ESLH, the only animal to display stimulus-bound drinking during testing was Animal 7.

Upon completion of testing, the nine animals which were not stimulus bound were given 7 more days of stimulation. All conditions were the same as those used in testing. Close observation showed that Animal 5 became a stimulus-bound wood gnawer, and Animal 8 consistently displayed gnawing or eating during the ESLH.

\section{DISCUSSION}

The purpose of this experiment was to ascertain whether the type of stimulus-bound behavior which initially emerges may be influenced by previous experience. The results showed that neither the experience with a consummatory response nor a strict contiguity between a response and ESLH was sufficient to influence the type of behavior which emerges.

These findings, taken together with those of Valenstein and Cox (1970), suggest that environmental variables do not typically play a major role in controlling the type of stimulus-bound response to initially emerge. This interpretation becomes more convincing when the usual screening procedure which lacks both specific previous experience with a response and a strict contiguity between the response and ESLH is considered.

Table 2

Mean Lick Rate Per Minute for the 5 Test Days as a Function of Stimulation Conditions

\begin{tabular}{ccc}
\hline Subject & Stimulation & No Stimulation \\
\hline 1 & 128 & 146 \\
2 & 142 & 134 \\
3 & 74 & 92 \\
4 & 62 & 92 \\
5 & 150 & 118 \\
6 & 86 & 104 \\
7 & 264 & 18 \\
8 & 140 & 170 \\
9 & 78 & 86 \\
10 & 140 & 168 \\
\hline
\end{tabular}


Although the prior experience did not affect the type of stimulus-bound behavior to emerge, it might have influenced the per cent of animals to display any type of stimulus-bound behavior. However, the 3 out of 10 animals to become stimulus bound did not differ from the 6 out of 20 ratio reported by Chisholm and Trowill (1976) and the 33\% stimulus-bound animals reported by Valenstein, Cox, and Kakolewski (1969).

Some recent reports have indicated that the magnitude of a previously established consummatory response in stimulus-bound animals is sensitive to the effects of deprivation, the volume of liquid ingested, and the palatability of the goal object (Chisholm \& Trowill, 1972; Mendelson, 1970; White, Wayner, \& Cott, 1970). These studies offer evidence showing that the consummatory response varies as a function of internal states and the nature of the goal object. However, this sensitivity does not appear to affect the type of response to initially emerge but only the magnitude of the response after it has already emerged.

\section{REFERENCES}

Chisholm, D. C., \& Trowill, J. A. The effects of incentive shifts on stimulus-bound licking in rats. Physiology and
Behavior, 1972, 9, 277-279.

Chisholm, D. C., \& Trowill, J. A. Consummatory responses as sources of reinforcement in electrically induced behaviors. Physiology and Behavior, 1976, 16, 51-53.

Mendelson, J. Food deprivation facilitates hypothalamic drinking. Physiology and Behavior, 1970, 5, 1225-1227.

Pellegrino, L. J., \& Cushman, A. J. A stereotaxic atlas of the rat brain. New York: Appleton-Century-Crofts, 1967.

Valenstein, E. S., \& Cox, V. C. Influence of hunger, thirst, and previous experience in the test chamber on stimulusbound eating and drinking. Journal of Comparative and Physiological Psychology, 1970, 70, 189-199.

Valenstein, E. S., Cox, V. C., \& Kakolewski, J. W. The hypothalamus and motivated behavior. In J. T. Tapp (Ed.), Reinforcement and behavior. New York: Academic Press, 1969. Pp. 242-283.

Valenstein, E. S., Cox, V. C., \& Kakolewski, J. W. Reexamination of the role of the hypothalamus in motivation. Psychological Review, 1970, 77, 16-31.

White, S. D., WAYNer, M. J., \& Cotr, A. Effects of intensity, water deprivation, prior water ingestion and palatability on drinking evoked by lateral hypothalamic electric stimulation. Physiology and Behavior, 1970, 5, 611-619.

(Received for publication November 7, 1975; revision accepted December 19, 1975.) 